\title{
Acute Septic Carpal Tunnel Syndrome in a Rock Climber
}

\section{Robert M Zbeda ${ }^{1}$, Remy V Rabinovich ${ }^{1}$, Mason Vialonga ${ }^{2}$, Daniel A Seigerman ${ }^{2}$}

\section{Learning Point of the Article:}

With the increasing popularity of indoor rock climbing gyms, clinicians should be aware of the possibility of developing a deep space hand infection.

\section{Abstract}

Introduction: Rock climbing is an increasingly popular sport in the United States. Acute and chronic upper extremity injuries related to rock climbing are frequently reported and include flexor pulley ruptures and hamate stress fractures. Deep space hand infections after indoor rock climbing are a sport-related pathology that has yet to be reported. Our purpose is to describe an acute septic carpal tunnel syndrome following rock climbing at an indoor climbing gym in a patient who required urgent irrigation and debridement.

Case Report: A 33-year-old Caucasian male presented with an acute septic carpal tunnel syndrome 4 days after rock climbing at an indoor gym. On physical examination, he exhibited numbness over the fingers, significant tenderness to palpation, and pain with passive range of motion. His inflammatory markers were markedly elevated and deep space hand infection was confirmed with computed tomography scans. The patient was taken for urgent exploration, irrigation and debridement, and carpal tunnel release.

Conclusion: We theorize that the patient had an abrasion on the finger or palm that created an entryway for a pathogen. We are unaware of another report of a deep space hand infection associated with rock climbing activities. This case report will hopefully spread awareness of this clinical entity to improve evaluation and prevention of hand infections in rock climbers, as well as providing guidelines for appropriate and timely treatment of the condition.

Keywords: Hand surgery, infection, rock climbing.

\section{Introduction}

Rock climbing is an increasingly popular athletic endeavor in the United States as demonstrated by the recent surge in participants and indoor climbing gyms [1]. The previous studies on rock climbing injury epidemiology have focused on chronic overuse injuries, fall from height, and acute traumatic injuries secondary to supraphysiologic loading. Flexor pulley ruptures and hamate stress fractures are some of the commonly reported injuries in the hand and wrist [2]. To the best of our knowledge, there is no report of severe hand infection directly related to indoor rock climbing.

We report on a patient who developed a deep space infection of the hand and acute septic carpal tunnel syndrome after rock climbing. Maintaining tight grips on climbing holds (shaped grips attached to climbing walls) can generate significant shear forces on the skin at the fingertips and palm. Moreover, it is customary for rock climbers to use chalk on their hands to improve grip. Abrasions and cuts may serve as potential entryways for pathogens and may result in pyogenic deep space infections and/or septic flexor tenosynovitis.

The purpose of our case report is to discuss a rare presentation of deep space hand infection and acute carpal tunnel syndrome following rock climbing at an indoor climbing gym that ultimately required urgent exploration, debridement, and decompression of the median nerve.

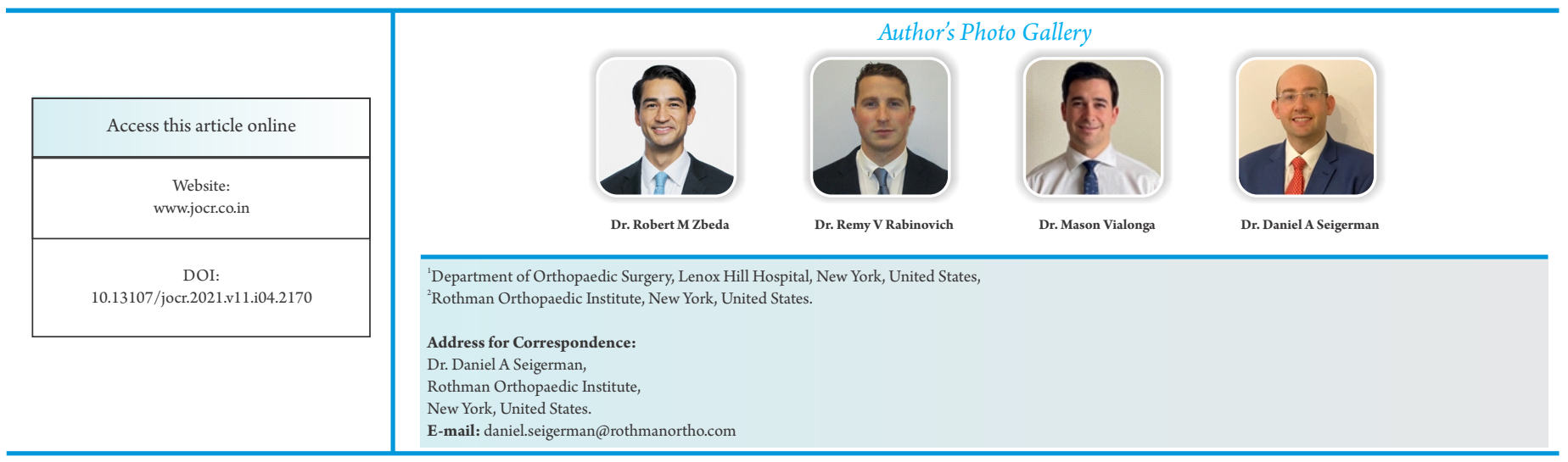

Journal of Orthopaedic Case Reports | pISSN 2250-0685 | eISSN 2321-3817 | Available on www.jocr.co.in | doi:10.13107/jocr.2021.v11.i04.2170 This is an Open Access article distributed under the terms of the Creative Commons Attribution Non-Commercial License (http://creativecommons.org/licenses/by-nc/3.0) which permits unrestricted non-commercial use, distribution, and reproduction in any medium, provided the original work is properly cited. 


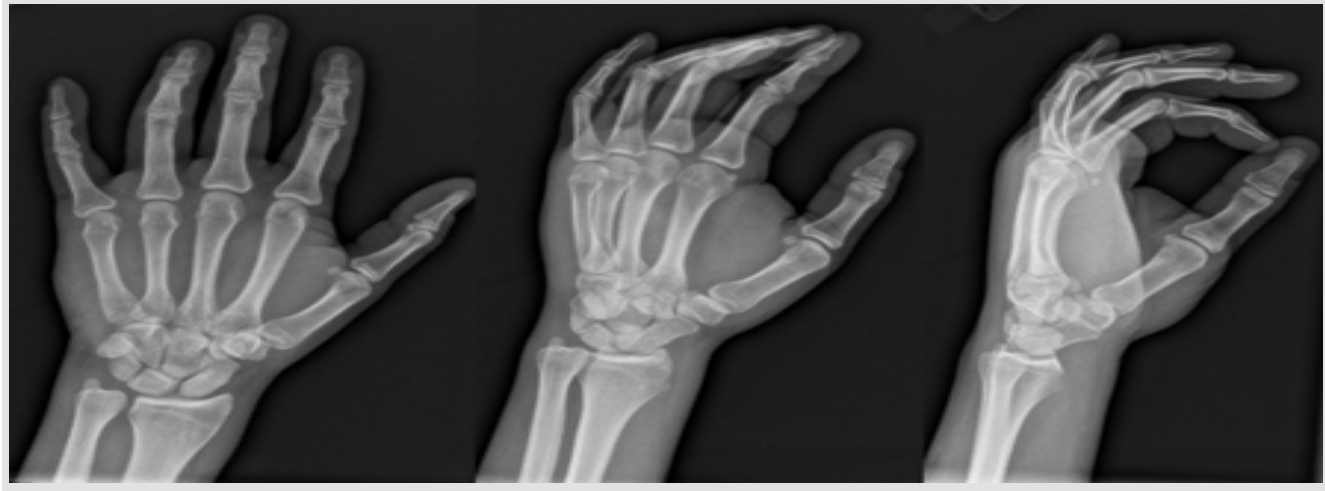

Figure 1: PA, oblique, and lateral radiograph of the left hand. There is soft-tissue swelling, but no fractures or foreign bodies noted.

Parona's space. Purulence was observed in all incisions and a large phlegmon was adherent to the median nerve (Fig. 3). The incisions were copiously irrigated and closed loosely. Culture was positive for Staphylococcus epidermidis. The patient had a course of 6 weeks of intravenous vancomycin and ceftriaxone and oral metronidazole under the direction of the infectious disease consultant. Sutures were removed

A 33-year-old, right hand dominant, male presented to the emergency department with 3 days of left hand pain. The patient went indoor rock climbing 4 days before arrival and began experiencing soreness in his left hand the following day. The patient subsequently developed worsening pain, erythema, and progressive numbness. He had no past medical or surgical history; no medications or allergies; and denied any use of alcohol, drugs, or tobacco.

On presentation, the patient was afebrile and vital signs were stable. The patient had pain with passive range of motion of his digits, maximal tenderness over the ring finger A1 pulley, and diminished sensation over the middle finger. His white blood cell count was 22,000, erythrocyte sedimentation rate (ESR) 22 $\mathrm{mm} / \mathrm{h}(\mathrm{nl}<15 \mathrm{~mm} / \mathrm{h})$, and C-reactive protein (CRP) 8.21 ( $\mathrm{nl}<$ $0.4 \mathrm{mg} / \mathrm{dL})$. Radiographs demonstrated soft-tissue swelling (Fig. 1). A computed tomography (CT) scan with IV contrast showed a rim-enhancing, fluid collection involving the flexor tendons at the level of the metacarpals (Fig. 2a, b). MRI was not readily available on an urgent basis.

The patient underwent urgent irrigation and debridement in the operating room. Incisions were made over the middle and ring finger A1 pulleys, mid-palmar space, carpal tunnel, and at 2 weeks and the patient began therapy (Fig. 4). The patient went on to fully resolve his infection and regained full range of motion, strength, and function of his left hand (Fig. 5).

\section{Discussion}

In 2017, an estimated 7.1 million people participated in rock climbing in the United States. Injuries from rock climbing are varied with $19-33 \%$ coming from chronic overuse, $28 \%$ from acute atraumatic supraphysiologic loading, and 10-39\% from a fall from height [2]. Rock climbing injuries can involve every part of the body, but occur frequently in the upper extremity [1]. Flexor pulley ruptures, hook of hamate stress fractures, and physeal stress fractures are climbing specific injuries occurring in the hand and wrist. As the popularity of rock climbing increases, the injuries associated with this sport will likely increase as well.

To date, there have been no reports of hand infections following rock climbing to our knowledge. Deep space infections and pyogenic flexor tenosynovitis (PFT) usually occur after a puncture wound. Acute carpal tunnel syndrome is defined by an increase in pressure exceeding $20-30 \mathrm{mmHg}$ within the carpal tunnel, which compromises epineurial blood flow and causes a

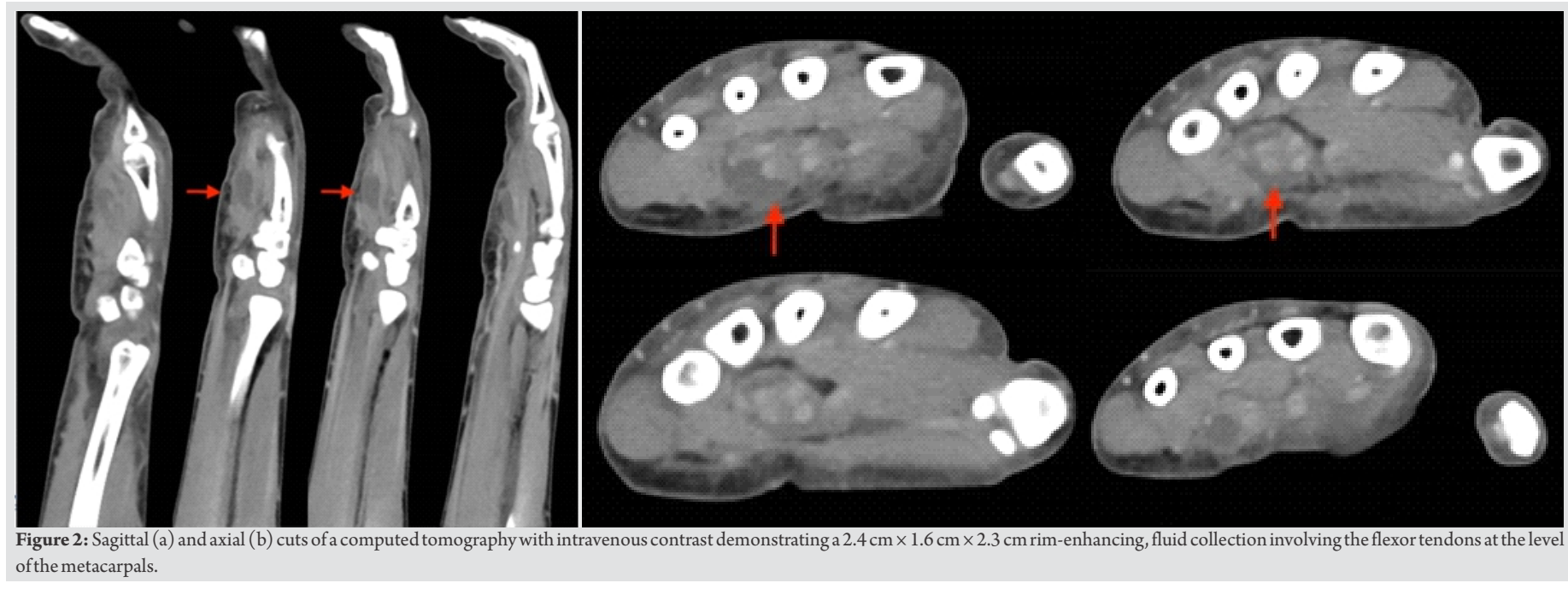




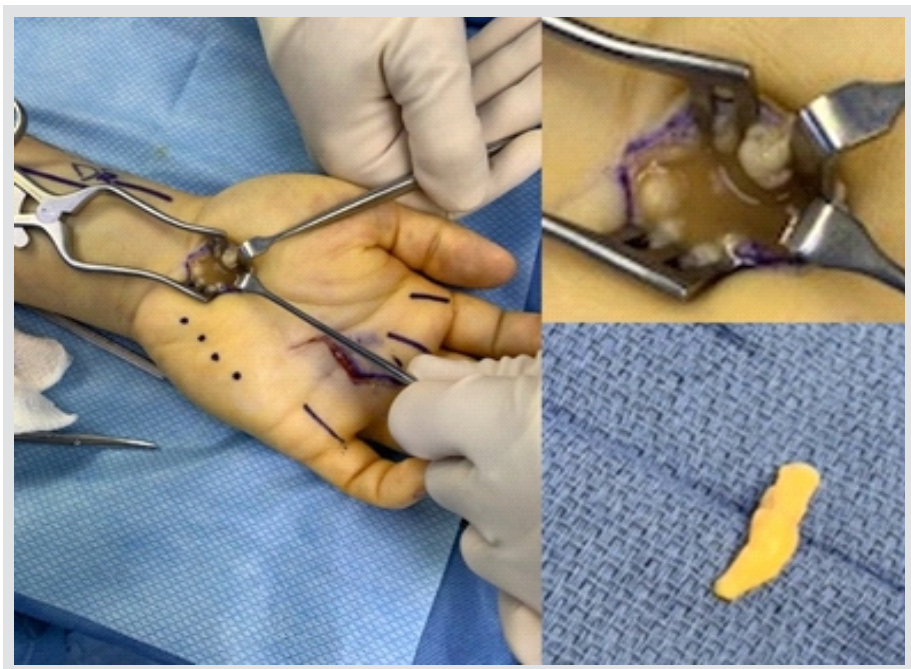

Figure 3: Intraoperative photographs of carpal tunnel release. There is gross purulence noted in the surgical site and a phlegmon was adherent to the median nerve and removed.

progressive worsening of median nerve symptoms [3]. Acute carpal tunnel syndrome secondary to pyogenic infection is a rare entity that has previously been reported in patients with animal bites, penetrating trauma, and indwelling central venous catheters $[3,4,5]$. We theorize that maintaining different hand grips on climbing holds can generate significant shearing forces at the fingertips and palm and may lead to abrasions and cuts. These fingertip abrasions are potential entry points for bacteria and continued contact between the potential entry points and the climbing surface provides more opportunity for infection to occur.

Proper management of hand infections in rock climbers should mirror that of hand infections in general. History and physical examination are paramount when evaluating patients for a potential deep space infection or PFT. Close examination of the hand may reveal wounds that are potential entryways for bacteria. Kanavel signs, points of maximal tenderness, and a thorough neurologic examination are important physical examination considerations. Positive Phalen test, Durkan test, and Tinel sign at the median nerve at the carpal tunnel, as well as numbness along the median nerve distribution are all suggestive of an acute carpal tunnel syndrome. Most important is to identify a patient who has rapidly progressive median nerve disturbance which becomes a surgical urgency as was noted in this case. Radiographs are important to rule out any bony trauma or foreign bodies. Advanced imaging, including CT, MRI, and ultrasound, may be helpful in the diagnosing hand infections, but is not always necessary and should not delay treatment if the clinical picture is highly suggestive of a deep infection. Laboratory tests to establish baselines for CRP and ESR may be helpful in evaluating the efficacy of treatments [6].

Patients with any neurologic compromise, as demonstrated in this case, should be taken urgently to the operating room for decompression as well as irrigation and debridement to prevent

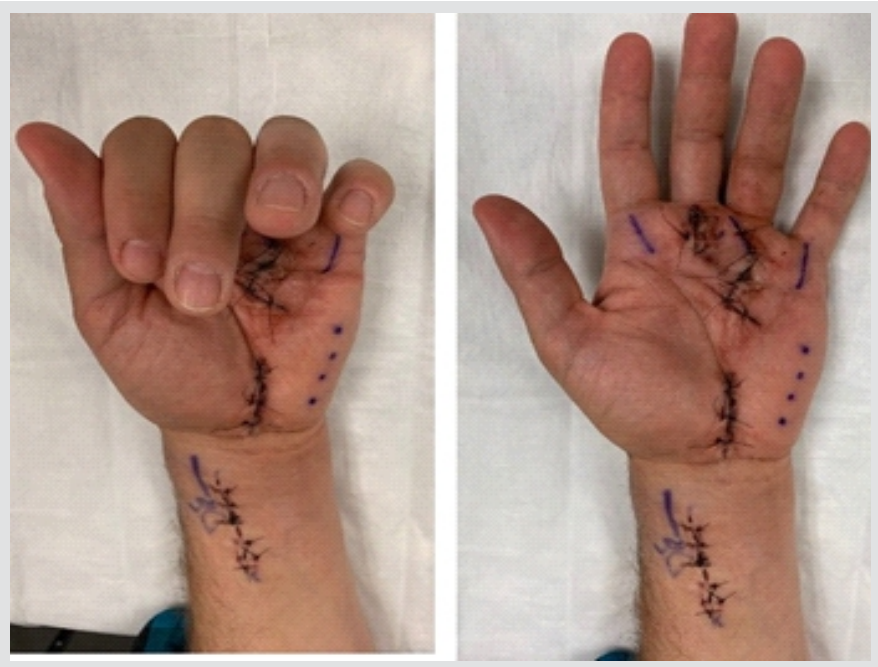

Figure 4: Clinical photograph at 1 week postoperatively. The sutures were removed and the patient was allowed to start range of motion exercises.

permanent neurologic injury. An understanding of the pathoanatomy of deep hand space infections is essential in performing an adequate debridement. The mid-palmar space or deep palmar space is bordered volarly by the flexor tendons and lumbricals, dorsally by the long and ring finger metacarpals, and second and third interossei, radially by the mid-palmar septum (septum of Legueu and Juvara), and ulnarly by the hypothenar muscles. The ulnar bursa commonly surrounds the flexor digitorum profundus and superficialis of the index, long, and ring fingers, but is not always contiguous with the tendon sheaths of these digits distally [6]. Proximally, the ulnar bursa and radial bursa can communicate through Parona's space in $80 \%$ of patients [7]. Parona's space is bordered by the pronator quadratus dorsally, digital flexor tendons volarly, flexor carpi ulnaris ulnarly, and flexor pollicis longus radially. This is clinically relevant as a deep infection in either bursa can lead to

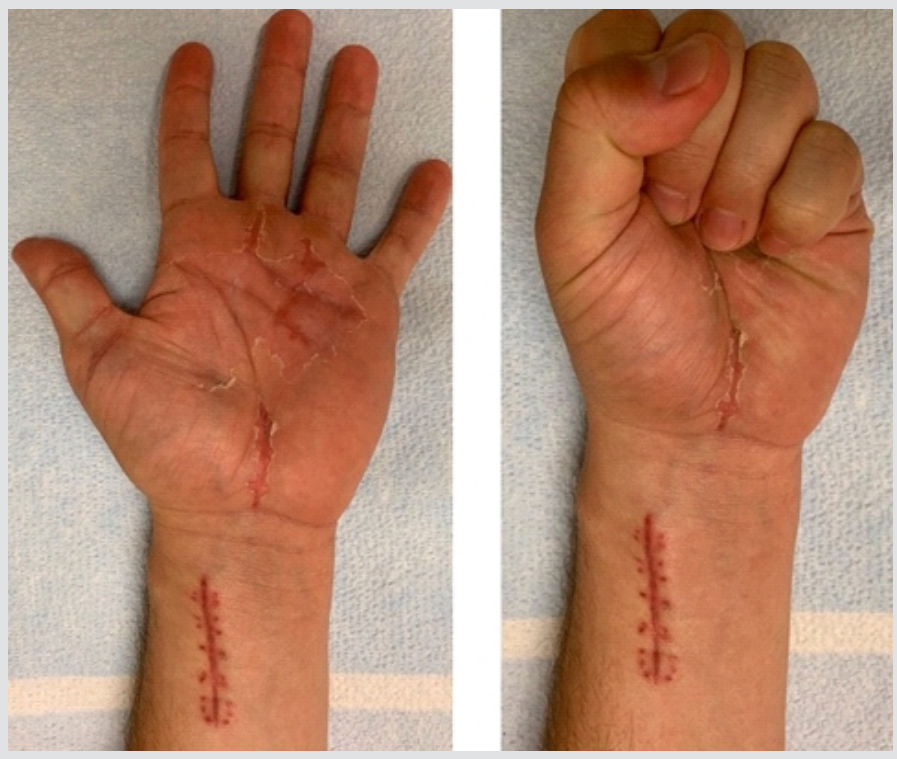

Figure 5: Clinical photograph at 4 weeks postoperatively. The incisions were well healed and the patient was able to regain near normal range of motion of the hand. 
horseshoe abscess and acute carpal tunnel syndrome may be present [8].

Antibiotic selection is dependent on the patient's history, comorbidities, organism encountered, and infection severity. Staphylococcus aureus infections account for up to $60 \%$ of hand infections [7]. Methicillin-resistant S. aureus (MRSA) is becoming increasingly prevalent in both the hospital and community setting. In severe infections, intravenous broadspectrum antibiotics, such as vancomycin and piperacillin/ tazobactam, can be initiated prophylactically pending the results of the wound cultures and antibiotic sensitivities. In cases of deep space hand infections, intravenous antibiotics for at least 3 weeks should be considered.

Proper hand hygiene and routine cleaning of climbing walls with disinfectant in indoor climbing gyms may help prevent infections in rock climbers. S. aureus and MRSA are prevalent in athletic training facilities and increase the risk of both local and systemic infections in athletes [9]. An infection control protocol involving introduction of disinfectant products and education may be effective in reducing bacteria, drug-resistant bacteria, and viral burden in training facilities. Through the introduction of an infection protocol in high school and collegiate training rooms, LaBelle et al. were able to reduce overall bacterial load by $94.7 \%$ and drug-resistant organisms (MRSA and vancomycin-resistant Enterococcus) and influenza by $100 \%$ [9]. Similar approaches applied in rock climbing facilities would likely be beneficial in reducing the

\section{References}

1. Nelson CE, Rayan GM, Judd DI, Ding K, Stoner JA. Survey of hand and upper extremity injuries among rock climbers. Hand (NY) 2017;12:389-94.

2. Cole KP, Uhl RL, Rosenbaum AJ. Comprehensive review of rock climbing injuries. J Am Acad Orthop Surg 2020;28:e501-9.

3. Brown BD, Roth TS, Gasner KA. Acute carpal tunnel syndrome after canine bite. J Am Acad Orthop Surg Glob Res Rev 2017;1:e069.

4. Flynn JM, Bischoff R, Gelberman RH. Median nerve compression at the wrist due to intracarpal canal sepsis. J Hand Surg Am 1995;20:864-7.

5. Sbai MA, Dabloun S, Benzarti S, Khechimi M, Jenzeri A, Maalla R. Acute carpal tunnel syndrome of the hand bacterial load as well. Other practices such as frequent hand washing, discouraging shared personal items, restricting individuals with open wounds or ongoing infections, and washing protective gear regularly are recommended practice in athletic groups [10]. In addition, climbers should maintain hand skin care, routinely check for wounds, and wear gloves or apply tape when necessary to prevent abrasions and cuts on theirhands.

\section{Conclusion}

The patient in this case report developed acute septic carpal tunnel syndrome after rock climbing at an indoor climbing gym. We suspect that the patient had an abrasion or cut on the finger or palm that created an entryway for a pathogen, resulting in a severe hand infection. We are unaware of another report of a hand infection associated with rock climbing activities. Our purpose is to spread awareness of this clinical entity to improve evaluation and prevention of hand infections in rock climbers, as well as providing guidelines for appropriate and timely treatment of the condition.

\section{Clinical Message}

With the increasing popularity of indoor rock climbing gyms, clinicians should be aware of the possibility of developing a deep space hand infection. Gym operators should maintain proper hygiene of the climbing walls and athletes should carefully monitor hand abrasions and wounds to protect themselves.

following a cat bite. Pan Afr Med J 2015;21:206.

6. Osterman M, Draeger R, Stern P. Acute hand infections. J Hand Surg Am 2014;39:1628-35.

7. Draeger RW, Bynum DK. Flexor tendon sheath infections of the hand.J Am Acad Orthop Surg 2012;20:373-82.

8. McDonald LS, Bavaro MF, Hofmeister EP, Kroonen LT. Hand infections. J Hand Surg Am 2011;36:1403-12.

9. LaBelle MW, Knapik DM, Arbogast JW, Zhou S, Bowersock L, Parker A, et al. Infection risk reduction program on pathogens in high school and collegiate athletic training rooms. Sports Health 2020;12:51-7.

10. Hostetter KS, Lux M, Shelley K, Drummond JL, Laguna P. MRSA as a health concern in athletic facilities. J Environ Health 2011;74:18-25.

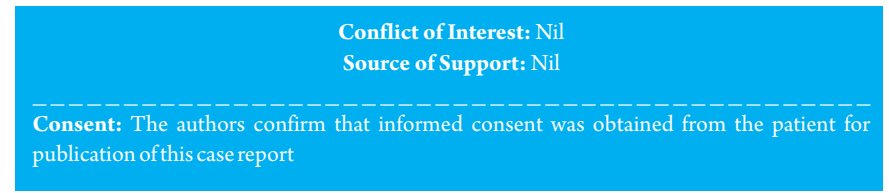

